Pacific Journal of 


\section{FOURIER TRANSFORMS AND THEIR LIPSCHITZ CLASSES}

\section{G. SAMPSON AND H. TUY}

We define a class of functions $A_{\alpha}$ for each $\alpha>0$. We show that the Fourier transform of every function of $A_{\alpha}$ exists and is Lipschitz of order $\alpha$. We construct examples to show that the converse is not true in general. However, we show that for a certain class of function $k$ (e.g., $k \in L_{2}$ ) if its Fourier transform $\hat{k}$ is Lipschitz of order $\alpha$ then $k \in A_{\beta}$ for all $\beta<\alpha$.

Boas ([1] and [2]) studied this problem in the case where the function $k$ is nonnegative and gave a complete solution in this case. In connection with this question several authors (e.g. Hirschman [5]; Liang Shin Hahn [4], Drobot, Naparstek and Sampson [3]) have proved mapping properties of convolution operators with kernel $k$, by studying the behavior of $\hat{k}$. To be more precise, they have proved mapping theorems when $\hat{k} \in \operatorname{Lip}(\alpha)$ with additional conditions on $k$. In our applications we prove a similar result (see $\$ 3$, Theorem 4).

Notations and Definitions.

$L_{\text {loc }}$ shall denote the set of all Lebesgue measurable functions integrable over all finite intervals. In this paper, the functions $f, g, k, \cdots \in L_{\text {loc }}$.

For $0<\alpha \leqq 1$, a function $f$ is Lipschitz of order $\alpha(f \in \operatorname{Lip}(\alpha))$ if there is a positive constant $A$ such that

$$
\sup _{x \in \mathbb{R}}|f(x+h)-f(x)| \leqq A|h|^{\alpha} .
$$

For $\alpha>1$, we say that a function $f$ is Lipschitz of order $\alpha$ if

(i) $f^{(m)} \in L_{\infty}$ for all $m<[\alpha]$ and

(ii) $f^{([\alpha])} \in \operatorname{Lip}(\alpha-[\alpha])$.

When we use the symbol

$$
\int_{a}^{b} g(t, x) d t \text { for } \quad-\infty \leqq a<b \leqq \infty .
$$

We are assuming that $g(t, x) \in L_{\text {loc }}$ as a function of $t$ for each $x$ and moreover the integral exists in the following sense:

$$
\int_{a}^{b} g(t, x) d t=\lim _{\substack{\alpha \rightarrow a \\ \beta \rightarrow b}} \int_{\alpha}^{\beta} g(t, x) d t .
$$


We write $h\left(x_{1}, x_{2}, \cdots, x_{n}, y\right)=O\left(y^{a}\right)$ to mean that there exists a positive number $C$ independent of $x_{1}, x_{2}, \cdots, x_{n}, y$ so that

$$
\sup _{\substack{x_{1} \in \mathbb{R} \\ 1 \leq n \leq n}}\left|h\left(x_{1}, x_{2}, \cdots, x_{n}, y\right)\right| \leqq C|y|^{a}
$$

In particular, we say $h\left(x_{1}, x_{2}, \cdots, x_{n}\right)=O(1)$ to mean that there exists a $C$ independent of $x_{1}, x_{2}, \cdots, x_{n}$ so that

$$
\sup _{\substack{x_{1} \in \mathbb{R} \\ \leq \leq n}}\left|h\left(x_{1}, x_{2}, \cdots, x_{n}\right)\right| \leqq C .
$$

We number each section independently.

\section{Sufficient conditions.}

LeMma 1. Let $a$ and $b$ be numbers so that $0<a<b$. Then for each $y>0$

$$
\begin{gathered}
\int_{0}^{y} t^{b} f(t, x) d t=O\left(y^{a}\right) \Leftrightarrow \int_{y}^{\infty} f(t, x) d t=O\left(y^{a-b}\right) \\
\int_{-y}^{0}|t|^{b} f(t, x) d t=O\left(y^{a}\right) \Leftrightarrow \int_{-\infty}^{-y} f(t, x) d t=O\left(y^{a-b}\right) .
\end{gathered}
$$

Proof. A similar lemma can be found in Boas's paper [1]. Thus we will be brief. We will prove (1); the argument for (2) is similar.

$\Rightarrow$ : Let $F(x, y)=\int_{0}^{y} t^{b} f(t, x) d t$, then we get

$$
\int_{y}^{\infty} f(t, x) d t=\left.t^{-b} F(x, t)\right|_{y} ^{\infty}+b \int_{y}^{\infty} t^{-b-1} F(x, t) d t .
$$

Since $F(x, y)=O\left(y^{a}\right)$ and also $a<b$ then we are through.

$\Leftarrow$ : Let $F(x, y)=\int_{y}^{\infty} f(t, x) d t$, then we get

$$
\int_{0}^{y} t^{b} f(t, x) d t=-\left.t^{b} F(x, t)\right|_{0} ^{y}+b \int_{0}^{y} t^{b-1} F(x, t) d t .
$$

Since $F(x, y)=O\left(y^{a-b}\right)$, then we are through.

LEMMA 2. Let $h>0$. 
(3)

$$
\text { If } \int_{1 / h}^{\infty} f(t, x) d t=O\left(h^{\alpha}\right), \text { then }
$$

$$
\int_{0}^{1 / h} f(t, x) \sin t h d t=O\left(h^{\alpha}\right) \text { for } \quad 0<\alpha<1
$$

$$
\int_{0}^{1 / h} f(t, x)(1-\cos t h) d t=O\left(h^{\alpha}\right) \text { for } 0<\alpha<2 .
$$

We get a similar result for $\int_{-\infty}^{-1 / h} f(t, x) d t$.

Proof. From Lemma 1 we get,

(6) $\int_{1 / h}^{\infty} f(t, x)=O\left(h^{\alpha}\right) \Leftrightarrow \int_{0}^{1 / h} t f(t, x) d t=O\left(h^{\alpha-1}\right)$ for $0<\alpha<1$.

$$
\Leftrightarrow \int_{0}^{1 / h} t^{2} f(t, x) d t=O\left(h^{\alpha-2}\right) \text { for } 0<\alpha<2
$$

To see this, it suffices to take $y=1 / h, a=b-\alpha$ where $b=1$ for (6) and $b=2$ for (7).

The function $\varphi(t)=(t h)^{-1} \sin$ th is decreasing and nonnegative for $t \in(0,1 / h)$. By the second-mean-value theorem for integrals we get,

$$
\int_{0}^{1 / h} t f(t, x) \varphi(t) d t=\int_{0}^{\xi} t f(t, x) d t \text { for some } \xi \in(0,1 / h) .
$$

Hence by hypothesis and (6) we conclude

$$
\int_{0}^{1 / h} t f(t, x) \varphi(t) d t=O\left(\xi^{1-\alpha}\right)=O\left(h^{\alpha-1}\right) .
$$

Consequently, we have

$$
\int_{0}^{1 / h} f(t, x) \sin t h d t=O\left(h^{\alpha}\right) .
$$

The proof for (5) is similar with $\varphi(t)=(t h)^{-2}(1-\cos t h)$.

Definition 3. Let $\alpha$ be a positive number. We say that $k \in A_{\alpha}$ if $k \in L_{\mathrm{loc}}$ and satisfies the following two conditions: 
(8)

$$
\int_{1 / h}^{\infty} f(t) e^{-t t x} d t=O\left(h^{\alpha}\right) \quad \text { and }
$$

$$
\int_{-\infty}^{-1 / h} k(t) e^{-1 t x} d t=O\left(h^{\alpha}\right)
$$

LEMMA 4. If $k \in A_{\alpha}$ then the Fourier transform $\hat{k}(x)=$ $(2 \pi)^{-1 / 2} \int_{-\infty}^{\infty} k(t) e^{-t t x} d t$ exists for all $x$ and $\hat{k} \in L_{\infty}$.

Proof. Since $k \in L_{\text {loc }}$ by (8) we get that $\hat{k}$ exists for each $x$. It also follows that $\hat{k} \in L_{\infty}$.

Lemma 5. Let $0<\alpha<1$. If $k \in A_{\alpha}$ then $\hat{k} \in \operatorname{Lip} \alpha \cap L_{\infty}$.

Proof. By Lemma 4, $\hat{k}$ exists for each $x$ and $\hat{k} \in L_{\infty}$.

Now we show that $\hat{k} \in \operatorname{Lip} \alpha$. Let $h>0$,

$$
\begin{aligned}
\hat{k}(x+h)-\hat{k}(x) & =(2 \pi)^{-1 / 2} \int_{-\infty}^{\infty} k(t) e^{-u x}\left(e^{-u h}-1\right) d t \\
& =(2 \pi)^{-1 / 2}\left\{\int_{-\infty}^{-1 / h}+\int_{-1 / h}^{1 / h}+\int_{1 / h}^{\infty}\right\} \\
\int_{-1 / h}^{0}+\int_{0}^{1 / h} & =O\left(h^{\alpha}\right) \text { by Lemma } 2 .
\end{aligned}
$$

By hypothesis we get,

$$
\int_{-\infty}^{-1 / h}+\int_{1 / h}^{\infty}=O\left(h^{\alpha}\right)
$$

Therefore, $\hat{k}(x+h)-\hat{k}(x)=O\left(h^{\alpha}\right)$.

We are going to show that the above lemma can be extended to $\alpha>1$ and $\alpha \notin \mathbf{N}^{+}\left(\mathbf{N}^{+}\right.$: set of positive integers). For $\alpha \in \mathbf{N}^{+}$we will give another sufficient condition. We are able also to give a sufficient condition on $k$ so that $\hat{k}$ is differentiable.

LEMMA 6. If $k$ satisfies

$$
\begin{aligned}
& \left|\int_{0}^{\infty} t k(t) e^{-u t x} d t\right|<\infty \quad \text { for each } x, \text { then } \\
& \lim _{h \rightarrow 0^{+}} \int_{0}^{1 / h} k(t) e^{-\imath t x} \frac{e^{-u h}-1}{h} d t=-i \int_{0}^{\infty} t k(t) e^{-t t x} d t \quad \text { for each } x .
\end{aligned}
$$


For the negative side we get a similar result.

Proof. It suffices to show

$$
\lim _{h \rightarrow 0^{+}} \int_{0}^{1 / h} t k(t) e^{-\imath t x} \frac{\sin t h}{t h} d t=\int_{0}^{\infty} t k(t) e^{-t t x} d t
$$

and

$$
\lim _{h \rightarrow 0^{+}} \int_{0}^{1 / h} t k(t) e^{-t t x} \frac{1-\cos t h}{t h} d t=0 .
$$

Let $\epsilon>0$ be given and let $x$ be such that (9) is true. Here, we keep $x$ fixed throughout the entire argument.

From (9), we conclude that there exists an $N>0$ and $0<h_{0}<1 / N$ such that for all $h$ satisfying $0<h<h_{0}$

$$
\left|\int_{N}^{1 / h} t k(t) e^{-u x} d t\right|<\epsilon
$$

Since the function $(1-\cos t h)(t h)^{-1}$ is monotonic and nonnegative in $(N, 1 / h)$ there exists a $\xi \in(N, 1 / h)$ so that

$$
\left|\int_{N}^{1 / h} t k(t) e^{-i t x} \frac{\cos t h-1}{t h}\right| \leqq\left|\int_{\xi}^{1 / h} t k(t) e^{-i t x} d t\right|
$$

It follows from (12) and (13) that

$$
\left|\int_{N}^{1 / h} t k(t) e^{-t t x} \frac{\cos t h-1}{t h} d t\right|<\epsilon
$$

On the other hand, since $t k(t) \in L_{\mathrm{loc}}$, by the Lebesque dominated convergence theorem (for $N$ fixed),

$$
\lim _{h \rightarrow 0^{+}} \int_{0}^{N} t k(t) e^{-i t x} \frac{\cos t h-1}{t h} d t=0 .
$$

Thus we get (11).

Now to show (10).

From (9), there exists $N_{0}$ such that for all $N$

$$
N>N_{0} \Rightarrow\left|\int_{0}^{\infty} t k(t) e^{-i t x} d t-\int_{0}^{N} t k(t) e^{-i t x} d t\right|<\epsilon .
$$


Since $(\sin t h)(t h)^{-1}$ is nonnegative and monotonic in $(N, 1 / h)$, then by a similar argument, there exists a fixed $N>N_{0}, h_{0}$ and $h_{1}<(1 / N)$ such that

$$
\begin{aligned}
& \left|\int_{N}^{1 / h} t k(t) e^{-t x} \frac{\sin t h}{t h} d t\right|<\epsilon \text { for all } 0<h<h_{0} \\
& \left|\int_{0}^{N} t k(t) e^{-t t x} \frac{\sin t h}{t h} d t-\int_{0}^{N} t k(t) e^{-t t x} d t\right|<\epsilon \\
& \text { for all } h \text { satisfying } 0<h<h_{1} .
\end{aligned}
$$

From (15), (16) and (17) we conclude that

$$
\begin{aligned}
\mid \int_{0}^{\infty} t k(t) e^{t t x} d t- & \int_{0}^{1 / h} t k(t) e^{-t t x} \frac{\sin t h}{t h} d t \mid
\end{aligned}
$$

Therefore we get (10) and we are through.

Theorem 7. Let $\alpha>1, m<\alpha$ and $m \in \mathbf{N}^{+}$. If $k \in A_{\alpha}$ then $\hat{k}$ is $m$ times differentiable at each $x$ and $\hat{k}^{(m)} \in L_{\infty}$. In fact

$$
\hat{k}^{(m)}(x)=(2 \pi)^{-1 / 2}(-i)^{m} \int_{-\infty}^{\infty} t^{m} k(t) e^{-t t x} d t
$$

Proof. By Lemma 1,

$$
\int_{1 / h}^{\infty} k(t) e^{-u x} d t=O\left(h^{\alpha}\right) \Rightarrow \int_{0}^{1 / h} t^{\alpha+1} k(t) e^{-u x} d t=O\left(h^{-1}\right) .
$$

Hence by Lemma 1 again, for all $m<\alpha$

$$
\int_{1 / h}^{\infty} t^{m} k(t) e^{-t t x} d t=O\left(h^{\alpha-m}\right)
$$

Thus $t^{m} k(t) \in A_{\alpha-m}$.

It follows from Lemma 4 that

$$
f_{m}(x)=(2 \pi)^{-1 / 2}(-i)^{m} \int_{-\infty}^{\infty} t^{m} k(t) e^{-u x} d t
$$

exists for each $x$ and $f_{m} \in L_{\infty}$. 
To prove the theorem, we first show that $\hat{k}^{\prime}(x)$ exists for each $x$. Since $k \in A_{\alpha}$, by Lemma $4 \hat{k}$ exists and we have,

$\frac{\hat{k}(x+h)-\hat{k}(x)}{h}$

$$
=(2 \pi)^{-1 / 2} h^{-1}\left\{\int_{-\infty}^{-1 / h} k(t) e^{-t t x}\left(e^{-t t h}-1\right) d t+\int_{-1 / h}^{0}+\int_{0}^{1 / h}+\int_{1 / h}^{\infty}\right\} .
$$

Since $k \in A_{\alpha}(\alpha>1)$ by (8) we have $\lim _{h \rightarrow 0} h^{-1}\left(\int_{1 / h}^{\infty}+\int_{-\infty}^{-1 / h}\right)=0$. By (19) and Lemma 6 with $m=1$, it follows that $\hat{k}^{\prime}(x)=f_{1}(x)$.

The theorem is then true for $m=1$. Now we suppose that the theorem is true up to $m-1$, i.e. $\hat{k}^{(m-1)}(x)=f_{m-1}(x)$.

$$
\frac{\hat{k}^{(m-1)}(x+h)-\hat{k}^{(m-1)}(x)}{h}=(-1)^{m-1} \frac{\hat{g}(x+h)-\hat{g}(x)}{h}
$$

where $g(t)=t^{m-1} k(t)$.

Since $\alpha-m+1>1$, the above argument starting with (19) can be applied to $g(t)$ and we get,

$$
\lim _{h \rightarrow 0^{+}} \frac{\hat{g}(x+h)-\hat{g}(x)}{h}=-i(2 \pi)^{-1 / 2} \int_{-\infty}^{\infty} \operatorname{tg}(t) e^{-i t x} d t .
$$

Thus $\hat{k}^{(m)}(x)=f_{m}(x)$.

THEOREM 8. Let $\alpha>0$ and $\alpha \notin \mathbf{N}^{+}$.

If $k \in A_{\alpha}$ then $\hat{k} \in \operatorname{Lip} \alpha \cap L_{\infty}$.

Proof. For $0<\alpha<1$, this is Lemma 5.

Now look at the case where $\alpha>1$. By Lemma $4, \hat{k}$ exists for each $x$ and $\hat{k} \in L_{\infty}$. Due to Theorem 7 we can conclude that for all $m \leqq[\alpha]$, $\hat{k}^{(m)}$ exists and $\hat{k}^{(m)} \in L_{\infty}$. Moreover

$$
\hat{k}^{([\alpha])}(x)=(-i)^{[\alpha]}(2 \pi)^{-1 / 2} \int_{-\infty}^{\infty} t^{[\alpha]} k(t) e^{-i \alpha x} d t
$$

From (19) we get

$$
\int_{1 / h}^{\infty} t^{[\alpha]} k(t) e^{-i x} d t=O\left(h^{\alpha-[\alpha]}\right)
$$


Hence $t^{[\alpha]} k(t) \in A_{\alpha-[\alpha]}$. It follows from Lemma 5 that

$$
\widehat{t^{[\alpha]} k(t)} \in \operatorname{Lip}(\alpha-[\alpha])
$$

Hence by (21) we get our result.

Theorem 9. Let $\alpha \in \mathbf{N}^{+}$. If $k \in L_{\mathrm{loc}}$ and satisfies

$$
\int_{0}^{\infty} t^{\alpha} k( \pm t) e^{\mp u x} d t=O(1)
$$

and

$$
\int_{1 / h}^{\infty} k( \pm t) e^{\mp t x} d t=o\left(h^{\alpha}\right)
$$

then $\hat{k} \in \operatorname{Lip} \alpha \cap L_{\infty}$.

Proof. First assume $\alpha=1$. By (23), $k \in A_{1}$. By Lemma 4, $\hat{k}$ exists for each $x$ and $\hat{k} \in L_{\infty}$. In (23) we are using the little "oo" notation. We note

$$
\frac{\hat{k}(x+h)-\hat{k}(x)}{h}=(2 \pi)^{-1 / 2} h^{-1}\left\{\int_{-\infty}^{-1 / h}+\int_{-1 / h}^{1 / h}+\int_{1 / h}^{\infty}\right\}
$$

Hence by Lemma 6 and (23) we get

$$
\hat{k}^{\prime}(x)=-i(2 \pi)^{-1 / 2} \int_{-\infty}^{\infty} t k(t) e^{-\imath t x} d t
$$

From (22) we conclude that $\hat{k}$ is absolutely continuous. Hence $\hat{k} \in$ Lip (1).

For the case $\alpha>1$, we use induction. The argument is similar to that given in Theorem 7 and will be omitted here.

2. Necessary conditions. We know that for each $\alpha(0 \leqq$ $\alpha<1$ ) there exists a function $g$ such that $\hat{g} \in \operatorname{Lip}(\alpha)$ but $g \notin A_{\alpha}$. We give this example in $\$ 4$. However, we have succeeded in showing that $\hat{k} \in \operatorname{Lip}(\alpha)$ implies $k \in A_{\beta}$ for all $\beta<\alpha$ with some other conditions placed on $k$. One of the conditions that $k$ must satisfy is the following:

$$
\int_{v / 2}^{v} k(w) e^{-t t w} d w=i(2 \pi)^{-1 / 2} \int_{-\infty}^{\infty} \frac{\hat{k}(2 x+t)-\hat{k}(x+t)}{x} e^{i v x} d x
$$


Condition (1) is merely Parseval's identity, however we have only been able to show (1) holds for a certain class of functions. This result appears in Lemma 3 . In the case where $k \in L_{p}(1 \leqq p \leqq 2)$ and $\hat{k}$ is continuous, we can show (1) holds (the argument is similar to that given in Lemma 3).

Another condition that appears is,

Definition 1. Let $\epsilon \geqq \dot{0}$. We say that $f \in V_{\epsilon}$ if there exists some constant $A$ such that

$$
\int_{|x| \geqq A} \frac{\lfloor f(2 x+t)-f(x+t) \mid}{|x|^{1-\epsilon}} d x=O(1) .
$$

It is obvious that if $f \in L_{p}$ then $f \in V_{\epsilon}$ for $\epsilon<1 / p$. Furthermore, all constant functions belong to $V_{\epsilon}$ for all $\epsilon \geqq 0$.

THEOREM 2. If some $0<\epsilon<1, \hat{k} \in \operatorname{Lip} \alpha \cap V_{\epsilon}(0<\alpha \leqq 1)$ and $k$ satisfies (1) then $k \in A_{\beta}$ for all $0<\beta<\alpha$.

Corollary. Let $k \in L_{p}$ for some $1<p \leqq 2$. If $\hat{k} \in \operatorname{Lip}(\alpha)$, then $k \in A_{\beta}$ for all $0<\beta<\alpha$.

REMARK. In the above corollary $\hat{k}$ is defined as usual in the $L_{q}$ sense $(1 / p+1 / q=1)$. We note that for $0<n \leqq v \leqq 2^{\prime} n$ $\int_{n}^{v} k(w) e^{-\imath t w} d w=\int_{n}^{2^{2} n}+\cdots+\int_{c v}^{v}$ where $1 / 2 \leqq c \leqq 1$. Next we note that a formula similar to (1) holds for the term

$$
\int_{c v}^{v} k(w) e^{-u t w} d w, \quad 1 / 2 \leqq c \leqq 1 .
$$

And now the Corollary follows.

Proof of Theorem 2. Let

$$
\varphi(x, t)=\frac{\hat{k}(2 x+t)-\hat{k}(x+t)}{x} .
$$

From (1) it follows for $v>1$ (note $k \in L_{\mathrm{loc}}$ )

$$
\begin{aligned}
& 2 \int_{v / 2}^{v} k(w) e^{-1 t w} d w \\
& \quad=\left(\int_{-\infty}^{-2 \pi / v}+\int_{-2 \pi / v}^{\pi / v}+\int_{\pi / v}^{\infty}\right)(\varphi(x, t)-\varphi(x+\pi / v, t)) e^{i v x} d x .
\end{aligned}
$$

For the middle term on the right hand side of (3) we note that there is some constant $C$ such that,

$$
|\varphi(x, t)| \leqq C|x|^{\alpha-1} \text { and }|\varphi(x+(\pi / v))| \leqq C|x+(\pi / v)|^{\alpha-1} .
$$


It fóllows that

$$
\int_{-2 \pi / v}^{\pi / v}=O\left(v^{-\alpha}\right)
$$

For the remaining terms we write,

(5) $\varphi(x, t)-\varphi(x+\pi / v, t)=\psi(x, v, t)+\pi / v\left(\frac{\hat{k}(2 x+t)-\hat{k}(x+t)}{x(x+\pi / v)}\right)$ where

$$
\psi(x, v, t)=\frac{\hat{k}(2 x+t)-\hat{k}(x+t)-\hat{k}(2 x+(2 \pi / v)+t)+\hat{k}(x+(\pi / v)+t)}{x+(\pi / v)} .
$$

To complete our argument we need to show that

$$
\left(\int_{-\infty}^{-2 \pi / v}+\int_{\pi / v}^{\infty}\right) \psi(x, v, t) e^{i v x} d x=O\left(v^{-\beta}\right) \forall \beta<\alpha
$$

The second term on the right hand side of (5) can be handled in a straightforward manner. We will give the argument for $\int_{-\infty}^{-2 \pi / v} \psi e^{i v x} d x$; the proof for $\int_{\pi / v}^{\infty} \psi e^{i v x} d x$ is similar. First let $\mu=\alpha / \epsilon$ and $s=$ $-v^{\mu}$. We get

$$
\left|\int_{-\infty}^{-2 \pi / v} \psi(x, v, t) e^{10 x} d x\right| \leqq\left(\int_{-\infty}^{s}+\int_{s}^{-2 \pi / v}\right)|\psi(x, v, t)| d x
$$

Since $\hat{k} \in \operatorname{Lip} \alpha$ we have

$$
|\psi(x, v, t)| \leqq C v^{-\alpha}|x+(\pi / v)|^{-1}
$$

for some constant $C$ independent of $x, v$, and $t$. It follows that

(7) $\int_{s}^{-2 \pi / v}|\psi(x, v, t)| d x \leqq C v^{-\alpha} \int_{s}^{-2 \pi / v} \frac{d x}{|x+(\pi / v)|}=O\left(v^{-\beta}\right) \forall \beta<\alpha$.

For the other term we have,

$$
\begin{aligned}
\int_{-\infty}^{s}|\psi(x, v, t)| d x \leqq & \int_{-\infty}^{s} \frac{|\hat{k}(2 x+t)-\hat{k}(x+t)|}{|x+(\pi / v)|^{1-\epsilon}|x+(\pi / v)|^{\epsilon}} d x \\
& +\int_{-\infty}^{s} \frac{|\hat{k}(2 x+(2 \pi / v)+t)-\hat{k}(x+(\pi / v)+t)|}{|x+(\pi / v)|^{1-\epsilon}|x+(\pi / v)|^{\epsilon}} d x .
\end{aligned}
$$


Since $\hat{k} \in V_{\epsilon}$ by the second mean value theorem for integrals we conclude that

$$
\int_{-\infty}^{s}|\psi(x, v, t)| d x=O\left(v^{-\alpha}\right)
$$

Hence the proof is complete.

LEMMA 3. If $k$ is a real valued function such that:

$$
\begin{gathered}
\hat{k} \quad \text { is continuous at } t \\
\int_{a}^{b} k(w) e^{-u w} d w=O(1)^{1} \quad \text { and } \\
\int_{|u| \geqq A}\left|\frac{\hat{k}(u)}{u}\right| d u<\infty \text { for some } A>0 .
\end{gathered}
$$

Then

$$
\int_{0 / 2}^{v} k(w) e^{-\imath w w} d w=i(2 \pi)^{-1 / 2} \lim _{\epsilon \rightarrow 0^{+}} \int_{|x| \geq \epsilon} \frac{\hat{k}(2 x+t)-\hat{k}(x+t)}{x} e^{i v x} d x .
$$

Proof. From (10) it follows that,

$$
\int_{\underline{a}}^{b} k(w) d w=O(1) \quad \text { and } \quad \hat{k} \in L_{\infty}
$$

We will assume $v>0$, the proof for $v<0$ is similar. Let $P_{\delta}(u)=$ $\delta /\left(\delta^{2}+u^{2}\right)$ which is the well-known Poisson kernel. We begin by showing that

$$
\int_{v / 2}^{\nu} k(u) e^{-t u} d u=\lim _{\delta \rightarrow 0^{+}} 1 / \pi \int_{v / 2}^{0} e^{-u t w} \int_{-\infty}^{\infty} k(u) P_{\delta}(w-u) d u d w .
$$

Using (12) we note that

$$
\lim _{\delta \rightarrow 0^{+}} \int_{v / 2}^{v} e^{i t w}\left(\int_{-\infty}^{v / 4}+\int_{2 v}^{\infty}\right) k(u) P_{\delta}(w-u) d u d w=0 .
$$

Hence since $k \in L_{\text {loc }}$ we get

\footnotetext{
${ }^{1}$ Refer to page 2 with $g(u, a, b)=O(1)$.
} 
530

G. SAMPSON AND H. TUM

$$
\begin{aligned}
\lim _{\delta \rightarrow 0^{+}} \int_{v / 2}^{v} e^{-\imath w} \int_{v / 4}^{2 v} k(u) P_{\delta}(w-u) d u d w & \\
& =\lim _{\delta \rightarrow 0^{+}} 1 / \pi \int_{v / 4}^{2 v} k(u) \int_{v / 2}^{v} e^{-u w} P_{\delta}(w-u) d w d u .
\end{aligned}
$$

Now (13) follows immediately.

By (10) and the second mean value theorem for integrals we get

$$
\int_{-\infty}^{\infty} e^{-\delta|u|} \hat{k}(u) e^{i w u} d u=2(2 \pi)^{-1 / 2} \int_{-\infty}^{\infty} k(u) P_{\delta}(w-u) d u .
$$

From (13) and (14) and using the fact that $\hat{k} \in L_{\infty}$ we get

$$
\begin{aligned}
\int_{v / 2}^{v} k(w) e^{u w} d w & =\lim _{\delta \rightarrow 0^{+}}(2 \pi)^{-1 / 2} \int_{v / 2}^{v} e^{-u t w} \int_{-\infty}^{\infty} e^{-\delta|u|} \hat{k}(u) e^{\imath w u} d u d w \\
& =\lim _{\delta \rightarrow 0^{+}}\left(\int_{|u-t| \leq \epsilon}+\int_{|u-t| \geq \epsilon}\right) e^{-\delta|u|} \hat{k}(u) \rho(u-t, v) d u
\end{aligned}
$$

where $\rho(u, v)=(2 \pi)^{-1 / 2}(i u)^{-1}\left(e^{u v v}-e^{u v / 2}\right)$.

We note that there exists some constant $C$ independent of $\delta$ and $\epsilon$ such that

$$
\left|\int_{|u-r| \leq \epsilon} e^{-\delta|u|} \hat{k}(u) \rho(u-t, v) d u\right| \leqq C \epsilon .
$$

By (11) we can conclude that,

$$
\lim _{\delta \rightarrow 0^{+}} \int_{|u-t| \geq \epsilon} e^{-\delta|u|} \hat{k}(u) \rho(u-t, v) d u=\int_{|u-t| \geq \epsilon} \hat{k}(u) \rho(u-t, v) d u .
$$

After substitution we have,

$$
\begin{aligned}
& \text { 17) } \quad i \int_{|u-t| \geq \epsilon} \hat{k}(u) \rho(u-t, v) d u \\
& =(2 \pi)^{-1 / 2}\left\{\int_{|x| \geq \epsilon} \frac{\hat{k}(x+t)-\hat{k}(2 x+t)}{x} e^{i v x} d x-\int_{\epsilon / 2 \leq|x| \leq \epsilon} \frac{\hat{k}(2 x+t)}{x} e^{i v x} d x\right\} .
\end{aligned}
$$

We have,

$$
\begin{aligned}
\left|\int_{\epsilon / 2 \leqq|x| \leqq \epsilon} \frac{\hat{k}(2 x+t)}{x} e^{i x x} d x \cdot\right| \\
\quad \leqq \int_{\epsilon / 2 \leqq|x| \leqq \epsilon} \frac{|\hat{k}(2 x+t)-\hat{k}(t)|}{|x|} d x+\left|\hat{k}(t) \int_{\epsilon / 2 \leqq|x| \leqq \epsilon} \frac{e^{v x}}{x} d x\right| .
\end{aligned}
$$


Since $\hat{k} \in L_{\infty}$ and $\hat{k}$ is continuous at $t$,

$$
\lim _{\epsilon \rightarrow 0^{+}} \int_{\epsilon \in 2 \leqq|x| \leq \epsilon} \frac{\hat{k}(2 x+t)}{x} e^{i v x} d x=0
$$

The conclusion follows from (15), (16), (17) and (18).

\section{Applications.}

1. If $k(t)=e^{|t| t^{\mid}} /\left(|t|^{b}+1\right)$ where $a(a-1) \neq 0$ and $b+a / 2-1>0$, then $\hat{k} \in \operatorname{Lip}(b+a / 2-1)$. This follows immediately from Theorem 8 of $\$ 1$, and van der Corput's Lemma (see [6]).

2. We adopt the following definitions:

Definition 1. We say that $k \in L_{p}^{p}$ if for all $f \in L_{0}^{\infty}$ (set of $L_{\infty}$ functions with compact support)

$$
\int|k * f|^{p} \leqq C \int|f|^{p}
$$

where $C$ is independent of $f$.

Definition 2. We say that $k \in L_{2}^{*}$ if for all $f \in L_{0}^{\infty}$, there is a constant $C$ such that

$$
\left|\left\{x:\left|\int_{0}^{\infty} k( \pm t) f(x \pm t) d t\right|>y\right\}\right| \leqq \frac{C}{y^{2}}\|f\|_{2}^{2} \text {, for all } y>0 \text {. }
$$

Here, $C$ is independent of $f$ and $y$.

Lemma 3. (Jurkat and Sampson). If $k \in L_{2}^{*}$ and $\int_{s}^{2 s}|k(t)| d t=$ $O(1)$ for all $s$, then $\int_{a}^{b} k(t) e^{-t t x} d t=O(1)$.

Proof. Let $f$ be the characteristic function of $[0,2 b]$ with $b>$ 0 . For all $u \in[0, b]$ we have, for fixed $x$,

$$
\int_{0}^{\infty} f(u+t) k(t) e^{-i t x} d t=\left(\int_{0}^{b}+\int_{b}^{2 b-u}\right) k(t) e^{-i x t} d t .
$$

But

$$
\left|\int_{b}^{2 b-u} k(t) e^{-i x t} d t\right| \leqq \sup _{s \in R}\left|\int_{s}^{2 s}\right| k(t)|d t|=M \text {. }
$$


Therefore if $\left|\int_{0}^{b} k(t) e^{-x t} d t\right| \leqq 2 M$ the proof is over. Now suppose that $\left|\int_{0}^{b} k(t) e^{-i x t} d t\right|>2 M$. In this case, from (1) it follows that

$$
\left|\left\{u:\left|\int_{0}^{\infty} k(t) e^{-u x t} f(u+t) d t\right|>\frac{1}{2}\left|\int_{0}^{b} k(t) e^{-i x t} d t\right|\right\}\right| \geqq b .
$$

Since $k \in L_{2}^{*}$, there exists some constant $C$ independent of $x$ and $b$ such that

$$
\left|\left\{u:\left|\int_{0}^{\infty} k(t) e^{-x(t+u)} f(u+t)\right|>\frac{1}{2}\left|\int_{0}^{b} k(t) e^{-i x t} d t\right|\right\}\right|
$$

$$
\leqq \frac{C}{\left|\int_{0}^{b} k(t) e^{-\mathrm{uxt}} d t\right|^{2}} \int_{0}^{\infty}|f(t)|^{2} d t
$$

From (2) and (3) it follows that $\left|\int_{0}^{b} k(t) e^{-x x t} d t\right| \leqq \sqrt{C}$ where $C$ is independent of $b$ and $x$. A similar argument works for $b<0$ and hence we get our result. tions:

THEOREM 4. If $k$ is real-valued and satisfies the following condi-

$$
\begin{gathered}
\int_{s}^{2 s}|k(t)| d t=O(1) \\
\hat{k} \in \operatorname{Lip} \alpha \quad \text { for some } 0<\alpha<1 \\
|x|^{\lambda} \hat{k}(x)=O(1) \text { for some } \lambda>0, \text { and } \\
k \in L_{2}^{*} .
\end{gathered}
$$

Then $k \in L_{p}^{p}$ for $1<p<\infty$.

Proof. By Lemma 3, (4) and (7) imply that $\int_{a}^{b} k(t) e^{-i x t} d t=O(1)$. By Lemma 3 in $\$ 2$ we can conclude that (1) in $\$ 2$ holds. Furthermore (6) implies that $\hat{k} \in V_{\epsilon}$ for some $\epsilon>0$. Hence due to Theorem 2 in $\$ 2$, $k \in A_{\beta} \forall \beta<\alpha$. The conclusion follows from [3, Theorem 1.17]. 


\section{Examples.}

Lemma 1. Let $l, m, a$ and $b$ be given numbers. Set $M=$ $\max (|l|,|m|), L=\min (|l|,|m|)$ and $V=\max (|a|,|b|)$. Then,

$$
\begin{gathered}
\left|\int_{a}^{b} \frac{\sin l u \cos m u}{u} d u\right|=O(1) \\
\left|\int_{-b}^{b} \frac{\sin l u e^{-\imath m u}}{u} d u\right|=O(1)
\end{gathered}
$$

if $M$ and $V$ are sufficiently large,

$$
\left|\int_{a}^{b} \frac{\sin l u}{u} e^{-\imath m u} d u\right|=O(\log V+L)
$$

Proof. We get (1) since $\left|\int_{c}^{d} \sin u / u d u\right| \leqq A$ where $A$ is a positive constant independent of $c$ and $d$; also, (2) follows immediately from (1). For (3) it suffices to dominate

$$
\left|\int_{a}^{b} \frac{\sin l u}{u} \sin m u d u\right|
$$

Since the expression (4) is even and symmetric in $l$ and $m$ we can assume w.l.o.g. that $0<l \leqq m$. Furthermore, we can assume that $0 \leqq a<b$ since the integrand is an odd function in $u$. Thus

$$
\begin{gathered}
\left|\int_{a}^{b} \frac{\sin l u}{u} \sin m u d u\right| \leqq l \text { if } 0 \leqq a<b<1, \\
\left|\int_{a}^{b}\right| \leqq\left|\int_{a}^{1}\right|+\left|\int_{1}^{b}\right| \leqq l+\log b \text { if } 0 \leqq a \leqq 1<b,
\end{gathered}
$$

and

$$
\left|\int_{a}^{b}\right| \leqq \log b \quad \text { if } \quad 1<a<b .
$$

Hence we get our result.

THEOREM 2. For each $0<\alpha<1$ there exists a function $g \in L_{p}$ $(1 \leqq p<\infty)$ such that $\hat{\mathrm{g}} \in \operatorname{Lip} \alpha$ but $g \notin A_{\alpha}$. 
Proof. It suffices to show that there exists a $g \in L_{p}$ such that $\hat{g} \in \operatorname{Lip}(\alpha)$ and for some sequences $\left\{h_{n}\right\} \rightarrow 0,\left\{x_{n}\right\}$, and $\left\{B_{n}\right\} \rightarrow \infty$ then

$$
\left|h_{n}^{-\alpha} \int_{1 / h_{n}}^{2 / h_{n}} g(t) e^{-t x} d t\right|>B_{n} \quad \text { for } \quad\left|x-x_{n}\right| \leqq 1 / a_{n} .
$$

Consider

$$
g(t)=\sum_{m=1}^{\infty} \frac{\sin \left(m\left(t-c_{m}\right)\right)}{m^{\gamma-1} a_{m}^{\alpha}\left(t-c_{m}\right)} \chi_{\left[a_{m}, b_{m}\right]}^{(t)}
$$

where $\gamma$ is a fixed positive integer $\geqq 3$ and $\gamma \geqq(1-\alpha)^{-1}$. Also,

$$
a_{m}=2^{m^{\gamma}}, \quad b_{m}=2 a_{m} \quad \text { and } \quad c_{m}=3 / 2 a_{m}
$$

and $\chi_{I}$ is the characteristic function of $I$.

To show that $\hat{g}(x)$ exists for each $x \in \mathbf{R}$, it suffices to show that $g \in L_{1}$.

$$
\begin{aligned}
\int_{-\infty}^{\infty}|g(t)| d t & \leqq \sum_{m=1}^{\infty} m^{1-\gamma} a_{m}^{-\alpha} \int_{a_{m}}^{b_{m}} \frac{\left|\sin \left(m\left(t-c_{m}\right)\right)\right|}{\left|t-c_{m}\right|} d t \\
& \leqq 2 \sum_{m=1}^{\infty} \frac{1+\log \left(m a_{m}\right)}{m^{\gamma-1} a_{m}^{\alpha}}<\infty
\end{aligned}
$$

Now we are going to show that $\hat{g} \in \operatorname{Lip} \alpha$ for $\gamma \geqq(1-\alpha)^{-1}$.

Given $h$ such that $2|h|<1$, there exists an $m$ so that,

$$
\begin{aligned}
& 1 / a_{m+1}<|h| \leqq 1 / a_{m} \\
\hat{g}(x+h)-\hat{g}(x)= & \sum_{l=1}^{m-1} l^{1-\gamma} a_{l}^{-\alpha} \int_{a_{l}}^{b_{l}} \frac{\sin l\left(t-c_{l}\right)}{t-c_{l}} e^{-t+x}\left(e^{-t h}-1\right) d t \\
& +m^{1-\gamma} a_{m}^{-\alpha} \int_{a_{m}}^{b_{m}} \frac{\sin m\left(t-c_{m}\right)}{t-c_{m}} e^{-i t x}\left(e^{-u t h}-1\right) d t \\
& +\left(\sum_{l=m+1}^{\infty} l^{1-\gamma} a_{l}^{-\alpha} \int_{a_{l}}^{b_{l}} \frac{\sin l\left(t-c_{l}\right)}{t-c_{l}} e^{-u(x+h)} d t\right. \\
& \left.\quad-\sum_{l=m+1}^{\infty} l^{1-\gamma} a_{l}^{-\alpha} \int_{a_{l}}^{b_{l}} \frac{\sin l\left(t-c_{l}\right)}{t-c_{l}} e^{-i t x} d t\right) \\
= & I_{1}+I_{2}+I_{3} .
\end{aligned}
$$

We are going to show that each term on the right hand side of (11) is $O\left(h^{\alpha}\right)$ for $\gamma \geqq(1-\alpha)^{-1}$ separately. 
After substituting $u=t-c_{l}$, by (2) and (10) $I_{3}=O\left(h^{\alpha}\right)$.

Since $0<h<1 / a_{m}$, by the second mean value theorem for integrals there exists $\xi_{l}\left(a_{l}<\xi_{l}<b_{l}\right)$ such that

$$
\left|I_{1}\right| \leqq \sum_{l=1}^{m-1} \frac{\sin b_{l} h}{l^{\gamma-1} a_{l}^{\alpha}}\left|\int_{\xi_{l}}^{b_{1}} \frac{\sin l\left(t-c_{l}\right)}{t-c_{l}} e^{-u t x} d t\right|
$$

After substitution $u=t-c_{l}$, by (3), (9) and (10) it follows

$$
\left|I_{1} / h^{\alpha}\right| \leqq C \sum_{l=1}^{m-1}\left(a_{l} h\right)^{1-\alpha} l^{1-\gamma}\left(\log a_{l}+l\right)=O(1)
$$

It remains to show $I_{2}=O\left(h^{\alpha}\right)$. By (2) we have,

$$
I_{2} / h^{\alpha}=O\left(\left(h a_{m}\right)^{-\alpha} m^{1-\gamma}\right)=O(1)
$$

if $\left(h a_{m}\right)^{-\alpha} m^{1-\gamma} \leqq 1$

Since $e^{-t t h}-1=(\cos t h-1)+i \sin t h$, by the second mean value theorem for integrals and (3) we get

$$
I_{2} / h^{\alpha}=O\left(m\left(a_{m} h\right)^{1-\alpha}\right)=O(1), \quad \text { if } \quad m\left(a_{m} h\right)^{1-\alpha} \leqq 1
$$

Hence by (12) and (13) we conclude that $I_{2}=O\left(h^{\alpha}\right)$ if $\gamma \geqq(1-\alpha)^{-1}$.

Now we are going to show that there exist some sequences $\left\{h_{n}\right\} \rightarrow 0$, $\left\{x_{n}\right\}$, and $\left\{B_{n}\right\} \rightarrow \infty$ such that

$$
\left|\frac{1}{h_{n}^{\alpha}} \int_{1 / h_{n}}^{2 / h_{n}} g(t) e^{-t x} d t\right|>B_{n} \quad \text { for } \quad\left|x-x_{n}\right| \leqq 1 / a_{n}
$$

Consider $h_{m}=1 / c_{m}$ and $x_{m}=m$.

$$
h_{m}^{-\alpha} \int_{1 / h_{m}}^{2 / h_{m}} g(t) e^{-t t x} d t=J_{1}+J_{2}
$$

where

$$
J_{1}=-\frac{i e^{i c_{m} x}}{h_{m}^{\alpha} m^{\gamma-1} a_{m}^{\alpha}} \int_{0}^{1 / 2 a_{m}} \frac{\sin m v}{v} \sin x v d v
$$

and

$$
J_{2}=\frac{e^{\kappa_{m} x}}{h_{m}^{\alpha} m^{\gamma-1} a_{m}^{\alpha}} \int_{0}^{1 / 2 a_{m}} \frac{\sin m v}{v} \cos x v d v
$$


From (1) of Lemma 1 we can conclude that $J_{2}=O(1)$. Hence it suffices to show that $\left|J_{1}(x)\right| \geqq m / 2$ if $\left|x-x_{m}\right| \leqq 1 / a_{m}$.

$$
\left|J_{1}\right|=\left(\frac{3}{2}\right)^{\alpha} m^{1-\gamma}\left|\int_{0}^{1}+\int_{1}^{\frac{1}{2} a_{m}}\right|
$$

It is clear that $(\gamma \geqq 3)$

$$
m^{1-\gamma}\left|\int_{0}^{1} \frac{\sin m v}{v} \sin x v d v\right|=O(1)
$$

On the other hand,

$$
\begin{aligned}
& 2 m^{1-\gamma} \int_{1}^{\frac{1}{2} a_{m}} \frac{\sin m v}{v} \sin x v d v \\
& \quad=m^{1-\gamma} \int_{1}^{\frac{1}{2} a_{m}}\left(\frac{\cos (m-x) v}{v}+\frac{\cos (m+x) v}{v}\right) d v .
\end{aligned}
$$

We can easily see that for these $x$ 's,

$$
m^{1-\gamma} \int_{1}^{\frac{1}{2} a_{m}} \frac{\cos (m+x) v}{v} d v=O(1)
$$

For the remaining term we note that $\cos u \geqq 1-u^{2} / 2$. Therefore for $x$ satisfying $|x-m| \leqq 1 / a_{m}$,

$$
\begin{aligned}
m^{1-\gamma}\left|\int_{1}^{\frac{1}{2} a_{m}} \frac{\cos (m-x) v}{v} d v\right| & \geqq m^{1-\gamma} \int_{1}^{\frac{1}{2} a_{m}}\left(\frac{1}{v}-(m-x)^{2} v\right) d v \\
& \geqq m^{1-\gamma}\left(\log a_{m} / 2-\frac{(m-x)^{2} a_{m}^{2}}{8}\right) .
\end{aligned}
$$

Since $(m-x)^{2} a_{m}^{2} \leqq 1$ we conclude that for $m$ sufficiently large $\left|J_{1}\right| \geqq m / 2$.

The proof is then complete.

\section{REFERENCES}

1. R. P. Boas, Jr., Fourier series with positive coefficients, J. Math. Analysis and Appl., 17 (1967), 463-483.

2. Lipschitz behavior and integrability of characteristic functions, Ann. Math. Stat., 38 PTI (1967), 32-36. 
3. V. Drobot, A. Naparstek and G. Sampson, $\left(L_{p}, L_{q}\right)$ mapping properties of convolution transforms, Studia Mathematica, LV (1975), 41-70.

4. Liang Shin Hahn, On multipliers of p integrable functions, Trans. Amer. Math. Soc., 128 (1967), 321-335.

5. I. I. Hirschman, Jr., On multiplier transformations, Duke Math. J., 26 (1959), 221-242.

6. A. Zygmund, Trigonometric series, 2nd Edit. Vol. I and II, Cambridge Univ. Press, N. Y. (1959).

Received January 12,1976 . We wish to thank the referee for his helpful comments.

SUNY-BUFFALO

BufFalo, NY 14214 



\section{PACIFIC JOURNAL OF MATHEMATICS}

\section{EDITORS}

RICHARD ARENS (Managing Editor)

University of California

Los Angeles, CA 90024

R. A. BeAumont

University of Washington

Seattle, WA 98105

C. C. MOORE

University of California

Berkeley, CA 94720
J. DUGUNDJI

Department of Mathematics

University of Southern California

Los Angeles, CA 90007

R. FINN AND J. MILGRAM

Stanford University

Stanford, CA 94305

\section{ASSOCIATE EDITORS}
E. F. BECKENBACH
B. H. NEUMANN
F. WOLF
K. YoshidA

\section{SUPPORTING INSTITUTIONS}

UNIVERSITY OF BRITISH COLUMBIA

UNIVERSITY OF SOUTHERN CALIFORNIA

CALIFORNIA INSTITUTE OF TECHNOLOGY

STANFORD UNIVERSITY

UNIVERSITY OF CALIFORNIA

UNIVERSITY OF HAWAII

MONTANA STATE UNIVERSITY

UNIVERSITY OF TOKYO

UNIVERSITY OF NEVADA

UNIVERSITY OF UTAH

NEW MEXICO STATE UNIVERSITY

OREGON STATE UNIVERSITY

UNIVERSITY OF OREGON

OSAKA UNIVERSITY

WASHINGTON STATE UNIVERSITY

UNIVERSITY OF WASHINGTON

AMERICAN MATHEMATICAL SOCIETY

The Supporting Institutions listed above contribute to the cost of publication of this Journal, but they are not owners or publishers and have no responsibility for its contents or policies.

Mathematical papers intended for publication in the Pacific Journal of Mathematics should be in typed form or offset-reproduced (not dittoed), double spaced with large margins. Underline Greek letters in red, German in green, and script in blue. The first paragraph or two must be capable of being used separately as a synopsis of the entire paper. Items of the bibliography should not be cited there unless absolutely necessary, in which case they must be identified by author and Journal, rather than by item number. Manuscripts, in duplicate, may be sent to any one of the four editors. Please classify according to the scheme of Math. Reviews, Index to Vol. 39. All other communications should be addressed to the managing editor, or Elaine Barth, University of California, Los Angeles, California, 90024.

100 reprints are provided free for each article, only if page charges have been substantially paid. Additional copies may be obtained at cost in multiples of 50 .

The Pacific Journal of Mathematics is issued monthly as of January 1966. Regular subscription rate: $\$ 72.00$ a year (6 Vols., 12 issues). Special rate: $\$ 36.00$ a year to individual members of supporting institutions.

Subscriptions, orders for numbers issued in the last three calendar years, and changes of address should be sent to Pacific Journal of Mathematics, 103 Highland Boulevard, Berkeley, California, 94708.

PUBLISHED BY PACIFIC JOURNAL OF MATHEMATICS, A NON-PROFIT CORPORATION

Printed at Jerusalem Academic Press, POB 2390, Jerusalem, Israel. 


\section{Pacific Journal of Mathematics}

\section{Vol. 75, No. $2 \quad$ October, 1978}

Susan Jane Zimmerman Andima and W. J. Thron, Order-induced

topological properties ................................... 297

Gregory Wade Bell, Cohomology of degree 1 and 2 of the Suzuki groups . . 319

Richard Body and Roy Rene Douglas, Rational homotopy and unique

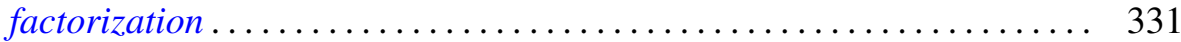

Frank Lewis Capobianco, Fixed sets of involutions ................. 339

L. Carlitz, Some theorems on generalized Dedekind-Rademacher sums .... 347

Mary Rodriguez Embry and Alan Leslie Lambert, The structure of a special class of weighted translation semigroups .....................

Steve Ferry, Strongly regular mappings with compact ANR fibers are

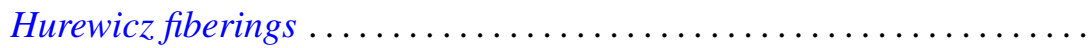

Ivan Filippenko and Marvin David Marcus, On the unitary invariance of the



$\mathrm{H}$. Groemer, On the extension of additive functionals on classes of convex

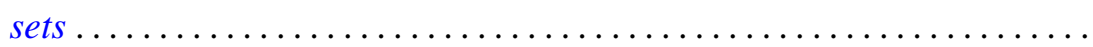

Rita Hall, On the cohomology of Kuga's fiber variety ............... 411

H. B. Hamilton, Congruences on $\mathrm{N}$-semigroups ................. 423

Manfred Herrmann and Rolf Schmidt, Regular sequences and lifting

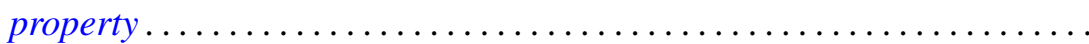

James Edgar Keesling, Decompositions of the Stone-Čech compactification which are shape equivalences .....................

Michael Jay Klass and Lawrence Edward Myers, On stopping rules and the expected supremum of $S_{n} / T_{n}$

Ronald Charles Linton, $\lambda$-large subgroups of $C_{\lambda}$-groups

William Owen Murray, IV and L. Bruce Treybig, Triangulations with the free cell property ............................

Louis Jackson Ratliff, Jr., Polynomial rings and $H_{i}$-local rings ...

Michael Rich, On alternate rings and their attached Jordan rings....

Gary Sampson and H. Tuy, Fourier transforms and their Lipschitz

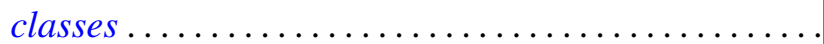

Helga Schirmer, Effluent and noneffluent fixed points on dendrites ...

Daniel Byron Shapiro, Intersections of the space of skew-symmetric maps

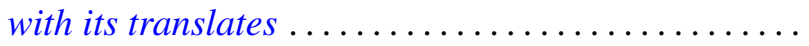

Edwin Spanier, Tautness for Alexander-Spanier cohomology ...

Alan Stein and Ivan Ernest Stux, A mean value theorem for binary digits ...

Franklin D. Tall, Normal subspaces of the density topology . .

William Yslas Vélez, Prime ideal decomposition in $F\left(\mu^{1 / p}\right) \ldots$ 